\title{
Investigation of Quantum Effects in Carbonaceous Materials Near the Metal-Insulator Transition by Means of THz Photoconductivity
}

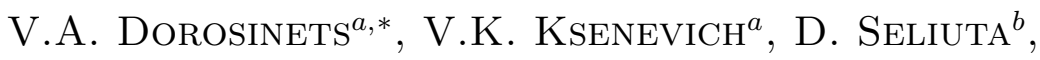 \\ Z. MARTŪNAS ${ }^{b}$ AND G. VALUŠIS ${ }^{b}$ \\ ${ }^{a}$ Belarussian State University, Nezaliezhnasti av.4, 220030 Minsk, Belarus \\ ${ }^{b}$ Semiconductor Physics Institute \\ A. Goštauto 11, LT- 01108 Vilnius, Lithuania
}

\begin{abstract}
A series of carbonaceous fibers with conductivity tuned to the metalinsulator transition were prepared by heat treatment of chemically modified polymer precursors. Peculiar behaviour of the resistivity versus temperature dependence $R(T)$ at low temperatures suggests quantum corrections to the Drude conductivity due to weak localization and electron-electron interaction dominating in the conductivity. The $\mathrm{THz}$ conductivity method is employed to study the modification of the density of states and provides evidence for a strong change in density of states at the Fermi level caused by the quantum effects.
\end{abstract}

PACS numbers: 71.30.+h, 72.15.Rn

\section{Introduction}

Carbonaceous materials provide an excellent "laboratory" to study quantum effects. In this work we focus on the investigation of the quantum effects in conductivity that approaches the metal-insulator transition (MIT) from the metallic side. This was achieved by introducing a randomness in the stacking of the graphenes and/or interlayer spacing, and the defects in individual graphenes. In the paper we study whether the $\mathrm{THz}$ photoconductivity can be used in monitoring a density of states (DOS) depletion at the Fermi level near the MIT due to quantum effects.

\section{Experimental}

We have developed a method for fabrication of carbon fibers that allows to vary the conductivity of the fibers in a controllable way in a wide range [1]. The

*corresponding author; e-mail: dorosinets@bsu.by 
method is based on the replacement of hydrogen ions by Co ions in COOH-group in a polymer matrix by means of ion-exchange absorption reaction. The following heat treatment in vacuum results in a formation of Co nanoparticles within the carbon matrix. The Co clusters catalyze structural ordering of amorphous carbon matrix driving the formation of graphenes at rather low temperatures, well below $1000^{\circ} \mathrm{C}$. By proper choice of the heat treatment temperature and the amount of the Co ions introduced we have managed to produce samples on insulating as well as on metallic sides of the MIT.

High-resolution transmission electron microscopy analysis has revealed that the Co clusters are well separated in the carbon matrix and hence the conductivity of the samples is determined only by structure of the carbon matrix itself for the samples investigated.

\section{Results and discussion}

We have studied three types of samples with conductivity close to the MIT. The data are presented in Fig. 1. Despite small change in the conductivity, the samples show strong difference in resistivity $(\mathrm{R})$-temperature $(T)$ dependence: the sample 1 shows a metal-like behaviour, the sample 2 shows a metal-like behaviour at high temperatures with transition at about $45 \mathrm{~K}$ to insulator-like behaviour (increase in resistivity with decrease in temperature), and the sample 3 shows an insulator-like behaviour. The change in sign of the $\mathrm{d} R / \mathrm{d} T$ dependence for the samples of the second group is suggestive of the appearance of the quantum effects that dominate the electronic transport at low temperatures. In general, the conductivity of disordered metals for which such quantum effects were observed can be described as [2]

$$
\sigma(T)=\sigma_{0}+\Delta \sigma_{\mathrm{WL}}(T)+\Delta \sigma_{\mathrm{EEI}}(T)
$$

where the terms $\Delta \sigma_{\mathrm{WL}}(T)$ and $\Delta \sigma_{\mathrm{EEI}}(T)$ represent quantum corrections to the Drude conductivity $\Delta \sigma_{0}$ and originate from weak localization (WL) and electronelectron interaction (EEI), respectively.

Analysis of the $\Delta \sigma(T)$ dependences for the samples of the third group reveals that they cannot be fitted by the laws that describe the hopping conductivity since they are on the metallic side of the MIT, despite of the unusual insulator-like shape of $R(T)$ dependence. We used a fitting procedure to the experimental data employing Eq. (1) to find quantum effects for this group.

For 3D case the localization correction term has the form $\Delta \sigma_{\mathrm{WL}} \sim T^{P / 2}$, where $P$ can attain the values of $1.5,2$, or 3 , depending whether the inelastic scattering rate is dominated by the electron-electron scattering in the dirty or clean limit, or by the electron-phonon scattering, respectively. For 2D case this dependence becomes logarithmic $\Delta \sigma_{\mathrm{WL}} \sim \ln (T)$. The interaction quantum correction term takes the form $\Delta \sigma_{\text {IEE }} \sim T^{1 / 2}$ and its magnitude depends on the electron screening factor. Among all possible combinations of the quantum correction terms only the expression 


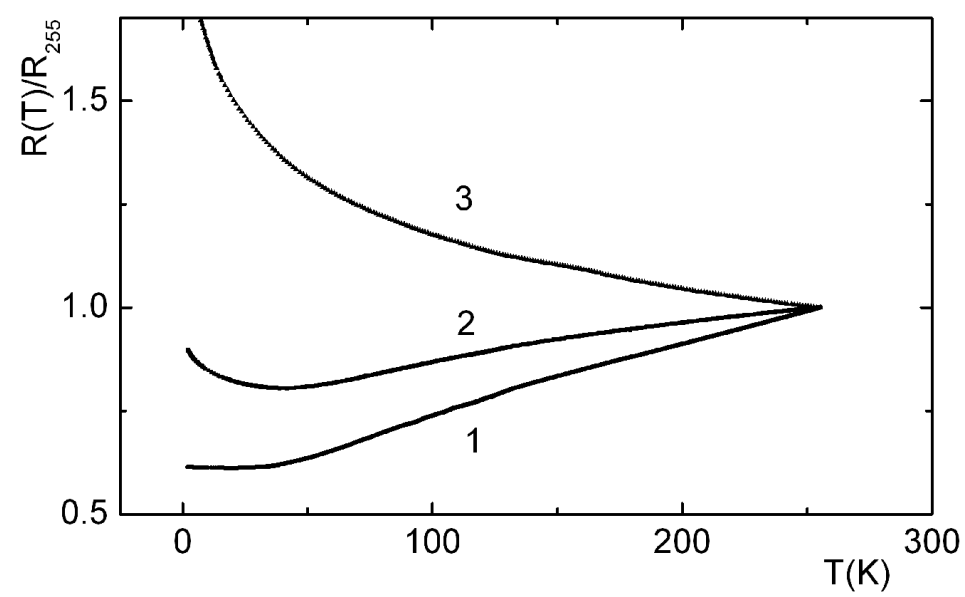

Fig. 1. Gradual change in shape of the $R(T)$ dependence for $\mathrm{C}(\mathrm{Co})$ fibers from classical metallic (1) to mixed (2) and further to insulator-like behaviour (3) with increasing disorder at the MIT transition.

$$
\sigma(T)=\sigma_{0}+A T^{1 / 2}+B \ln (T)
$$

where $A$ and $B$ are constants, shows an excellent fit to the experimental data in a wide temperature range. As Eq. (2) suggests, the electron conductivity is characterized by $3 \mathrm{D}$ EEI effect and 2D WL effects. The 2D WL effect for 3D systems, such as $\mathrm{C}(\mathrm{Co})$ fibers investigated, is not typical. This can be explained by layered structure of the pregraphitic carbon. The difference in magnitude of the Thouless length, characterizing the localization correction, and the Coulomb interaction characteristic length accounts for the difference in dimensionality of the two quantum effects [3].

The EEI quantum effect has been extensively studied in disordered metals and is known to reduce the density of states at the Fermi level, a phenomenon known as zero bias anomaly. Till now there is no enough understanding of how the WL quantum effect modifies the DOS.

The method of $\mathrm{THz}$ photoconductivity was used to prove the decrease in DOS caused by the quantum effects in the samples investigated in the low temperature range. The Joule heating of samples due to absorption of $2.5 \mathrm{THz}$ laser radiation diminishes the sensitivity of the measurements in some of the samples. To separate both effects we used the sample from the third group with a kink at about $45 \mathrm{~K}$ in the $R(T)$ curve. This kink is typical of intercalated graphite with enhanced disorder and is attributed to structural phase transitions. Figure 2 presents the $R(T)$ dependence of this samples as well as the corresponding photovoltage caused by THz radiation. Pronounced changes in the photoconductivity have been observed in the temperature range where the kink in the $R(T)$ dependence appears. The photoconductivity does not follow the resistance with increasing temperature but reveals strong fluctuation in magnitude near the kink. The onset temperature 


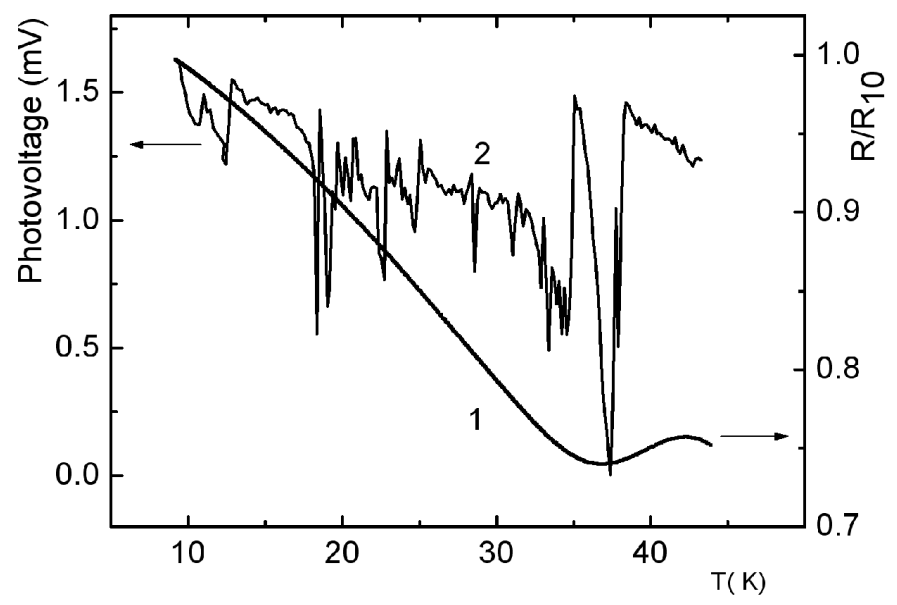

Fig. 2. $R(T)$ dependence of a $\mathrm{C}(\mathrm{Co})$ fiber of the type 3 and corresponding photovoltage induced by $\mathrm{THz}$ radiation.

of the fluctuations in the photoconductivity, the magnitude of the signal as well as its width does not directly correlate with the $R-T$ features in this temperature range. All this allows us to conclude that the $\mathrm{THz}$ conductivity signal reflects the strong changes in the DOS at the Fermi level due to quantum effects near the MIT.

\section{Conclusions}

We have shown that $\mathrm{THz}$ photoconductivity method has a great potential in investigation of the effects in disordered metals, where the quantum effects modify the DOS and allow to gain the insight into the nature of not yet well investigated processes which accompany the MIT.

\section{Acknowledgments}

This work was partly supported by NATO Collaborative Linkage grant (reference CBP.EAP.CLG982007) and Lithuanian State Science and Studies Foundation under contract V-07018.

\section{References}

[1] I. Bashmakov, V. Dorosinets, M. Lukashevich, A. Mazanik, T. Tihonova, T. Zabel, B. Wiedenhorst, H. Micklitz, J. Mater. Res. 16, 2832 (2001).

[2] P.A. Lee, T.V. Ramakrishnan, Rev. Mod. Phys. 57, 287 (1985).

[3] G. Du, V.N. Prigodin, A. Burns, J. Joo, C.S. Wang, A.J. Epstein, Phys. Rev. B 58, 4485 (1998). 